\title{
Impact of Fock terms on the isospin properties of nuclear matter
}

\author{
Bao Yuan Sun, Qian ZhaO and Wen Hui Long
}

\author{
${ }^{1}$ School of Nuclear Science and Technology, Lanzhou University, Lanzhou \\ 730000, People's Republic of China \\ ${ }^{2}$ Key Laboratory of Special Function Materials and Structure Design of \\ Ministry of Education, Lanzhou 730000, People's Republic of China
}

\begin{abstract}
Several topics on the isospin properties of nuclear matter studied within the density-dependent relativistic Hartree-Fock theory are summarized. In detail, the effects of the Fock terms on the nuclear symmetry energy are listed, including the extra enhancement from the Fock terms of the isoscalar meson-nucleon coupling channels, the extra hyperon-induced suppression effect originating from the Fock channel, self-consistent tensor effects embedded automatically in the Fock diagrams, the enhanced density-dependent isospin-triplet potential part of the symmetry energy at high densities, a reduced kinetic symmetry energy at supranuclear density and so on. The results demonstrate the importance of the Fork diagram, especially from the isoscalar mesonnucleon coupling channels, on the isospin properties of the in-medium nuclear force.
\end{abstract}

The isospin properties of nuclear matter, such as the nuclear symmetry energy, the nucleon isovector (symmetry) potential, and the neutron-proton effective mass splitting, play essential roles in studying several topics of nuclear structure, nuclear reactions and nuclear astrophysics [1-7]. Among them of great contemporary interest is the nuclear symmetry energy $E_{S}$ [8], which has been investigated extensively within both phenomenological approaches and microscopic many-body theories using almost all available effective and/or realistic nuclear interactions, see, e.g., Refs. [9-14]. Due 
to our limited knowledge about the in-medium effects of nucleon-nucleon $(N N)$ interactions and correlations along with technical difficulties to treat quantum many-body systems accurately, distinct deviations of predicted $E_{S}$ values appear beyond the nuclear saturation density $\rho_{0}$.

For the description of the structural properties of nuclear systems, the covariant density functional (CDF) theory has achieved great success during recent decades [15-23]. One of the most popular representatives is the relativistic Hartree approach with the no-sea approximation, namely, the relativistic mean field (RMF) theory [15,24-26]. However, due to the limit of the approach itself, significant system degrees of freedom are missing in RMF, such as the one-pion exchange. The non-local effects of nuclear forces and the momentum dependence of self-energies are also hardly to be treated. With the growth of computational facilities and the development of new methods, such defects can be eliminated with the inclusion of exchange (Fock) terms, which generates a new CDF model - the relativistic Hartree-Fock (RHF) theory with density-dependent meson-nucleon couplings (i.e., the DDRHF theory) [27]. Substantial improvements are gained by DDRHF in the selfconsistent description of the ground state properties [27-38], the excitation modes [39-42] of finite nuclei and nuclear astrophysics as well [43-46].

It has been shown from several studies that the inclusion of the Fock terms in the CDF theory could improve descriptions of the isospin-related nuclear structure. In fact, non-locality of the mean field induced by the Fock terms gives rise to the momentum dependence of the RHF self-energies, improving the isospin and energy dependence for the nucleon effective mass [27]. Besides the Fock terms in the isovector channel, those in the isoscalar channel are found also to play a dominant role in the evolution of the shell structure and the spin-orbit splitting [30]. Moreover, it has been demonstrated that the isoscalar Fock terms are essential for self-consistent description of the spin-isospin resonances like the GT and SD ones within RPA $[39,41]$ and the prediction of neutron star properties [43].

In this proceeding, we will give a short review about the impact of Fock terms on the isospin properties of nuclear matter within the DDRHF theory, in particular, its influence on the symmetry energy. The detailed discussion on these topics refers to Refs. [27, 43, 44, 46, 47].

The general formulae of the DDRHF theory in nuclear matter can be found in Refs. [27, 43, 46-48]. In RHF theory, the energy density functional (EDF) is obtained by taking the expectation value of the Hamiltonian with respect to the Hartree-Fock ground state, which consists of three parts: the kinetic EDF $\varepsilon_{k}$, the direct (Hartree) $\varepsilon_{\phi}^{D}$ and the exchange (Fock) $\varepsilon_{\phi}^{E}$ terms of the potential EDF. The meson- $N N$ interaction vertices consist of various 
meson-nucleon coupling channels, namely, the Lorentz scalar $(\sigma-\mathrm{S})$, vectors $(\omega-\mathrm{V}, \rho-\mathrm{V})$, vector-tensor $(\rho-\mathrm{VT})$, tensor $(\rho-\mathrm{T})$ and pseudovector $(\pi-\mathrm{PV})$ couplings. In DDRHF, the relativistic mass-energy relation has the form $E^{* 2}=M^{* 2}+\boldsymbol{p}^{* 2}$, where the starred quantities are defined as

$$
M^{*}=M+\Sigma_{S}(p), \quad \boldsymbol{p}^{*}=\boldsymbol{p}+\hat{\boldsymbol{p}} \Sigma_{V}(p), \quad E^{*}=E-\Sigma_{0}(p),
$$

where $\Sigma_{S}$ is the scalar self-energy, and $\Sigma_{0}$ and $\Sigma_{V}$ correspond to the time and space components of the vector self-energy, respectively. $M^{*}$ is also called the scalar mass or Dirac mass in several literatures. As a result, the binding energy per nucleon $E_{b}$ in nuclear matter for a given baryonic density $\rho_{b}$ and isospin asymmetry $\delta \equiv\left(\rho_{n}-\rho_{p}\right) / \rho_{b}$ can be obtained, and correspondingly the density-dependent symmetry energy is deduced from the second order coefficient of its Taylor expansion as

$$
E_{S}\left(\rho_{b}\right)=\left.\frac{1}{2} \frac{\partial^{2} E_{b}\left(\rho_{b}, \delta\right)}{\partial \delta^{2}}\right|_{\delta=0}=E_{S, k}+E_{S}^{D}+E_{S}^{E},
$$

including the kinetic part $E_{S, k}$, the Hartree $E_{S}^{D}$ and Fock $E_{S}^{E}$ potential parts.

The isospin dependence of the effective masses has been studied by defining its non-relativistic $\left(M_{N R}^{*}\right)$ and relativistic $\left(M_{R}^{*}\right)$ versions in the DDRHF theory [27]. As the Fock terms bring non-local effects into the RHF selfenergies, the isospin splitting of the nucleon effective mass in RHF calculations is significantly different from those in RMF. It is revealed that for the effective masses $M_{N R}^{*}$ at the corresponding Fermi energy $E_{F}$, the trend in RHF is that $M_{N R, n}^{*}\left(E_{F, n}\right)>M_{N R, p}^{*}\left(E_{F, p}\right)$ at lower density but reversed when approaching to the saturation density.

The DDRHF theory has then been utilized to study the symmetry energy and neutron star properties. The inclusion of the Fock terms strongly affects the density-dependence of the symmetry energy at high densities, and in turn the radius and cooling process of neutron stars [43,44,46,47]. Especially, it is found that not only the isovector mesons but also the isoscalar mesons can contribute to the symmetry energy with the inclusion of the Fock diagrams of meson-nucleon couplings [43]. In fact, the isoscalar mesons via the Fock terms provide more significant contributions to the symmetry energy than the isovector ones in the high-density region.

Motivated by recent observational data, the equations of state with the inclusion of $\Lambda$ hyperons and the corresponding properties of neutron stars are studied based on the DDRHF theory as well [44]. Because of the extra suppression effect originating from the Fock channel, large reductions 
induced by the occurrence of $\Lambda$ hyperons on the symmetry energy are predicted at high densities, resulting in a relatively small predicted value of the neutron-star radius.

In addition, with the possibility to extract the nuclear tensor interaction directly from the Fock diagrams of various meson-nucleon couplings [38], distinct tensor effects are illustrated in the exploration of the isospin properties of nuclear matter and neutron star structures [46]. The naturally involved tensor force components in the Fock diagrams soften the density-dependent behavior of the symmetry energy and consequently lead neutron stars to be more compact. Then the threshold density for the direct Urca process that cools the neutron star rapidly is raised by the nuclear tensor force.

To clarify the isospin structure of in-medium $N N$ interaction, the isospin coupling-channel decomposition of the symmetry energy is carried out within the DDRHF theory [47]. When the Fock terms is involved, it is found that both isospin-singlet and isospin-triplet components of the potential energy play an important role in determining the symmetry energy. A strong density-dependent behavior is revealed in the isospin-triplet potential part of the symmetry energy at high densities. The results demonstrate again the importance of the Fork diagram, especially from the isoscalar-meson coupling channels, on the isospin properties of the in-medium nuclear force.

Recently, the kinetic EDF $\varepsilon_{k}$ has been identified as a particularly good indicator of the short range correlations due to the tensor force in the nuclear ground state [49-52]. It is found that the contribution from $\varepsilon_{k}$ to the symmetry energy is strongly reduced and even becomes negative due to such correlations when compared to the non-interacting case [50-52]. In CDF theory, the kinetic EDF $\varepsilon_{k}$, which contains the self-energies, gets the in-medium $N N$ interaction involved as well [43].

$$
\varepsilon_{k}=\frac{1}{\pi^{2}} \sum_{i=n, p} \int_{0}^{k_{F, i}} M \hat{M} p^{2} d p+\frac{1}{\pi^{2}} \sum_{i=n, p} \int_{0}^{k_{F, i}} p \hat{P} p^{2} d p \equiv \varepsilon_{k}^{M}+\varepsilon_{k}^{P},
$$

where $\hat{M}=M^{*} / E^{*}, \hat{P}=\boldsymbol{p}^{*} / E^{*}$, and $\varepsilon_{k}^{M}$ and $\varepsilon_{k}^{P}$ denote the contributions from the nucleon mass and momentum, respectively. The hatted quantity $\hat{M}$ could be further separated into

$$
\hat{M}=\frac{M}{E^{*}}+\frac{\Sigma_{S}^{D}}{E^{*}}+\sum_{\phi} \frac{\Sigma_{S}^{E, \phi}}{E^{*}} .
$$

Thus, the in-medium effects hidden in $\varepsilon_{k}$ could be investigated in detail within the CDF theory, in particular the contribution due to the short range 
correlations [47]. It is found that the inclusion of the Fock terms in DDRHF causes a sizable reduction on the kinetic part $E_{S, k}$ of the symmetry energy, as compared to the RMF results [47]. At supranuclear density region, the DDRHF results even become negative, as seen in the left panel of Fig. 1.
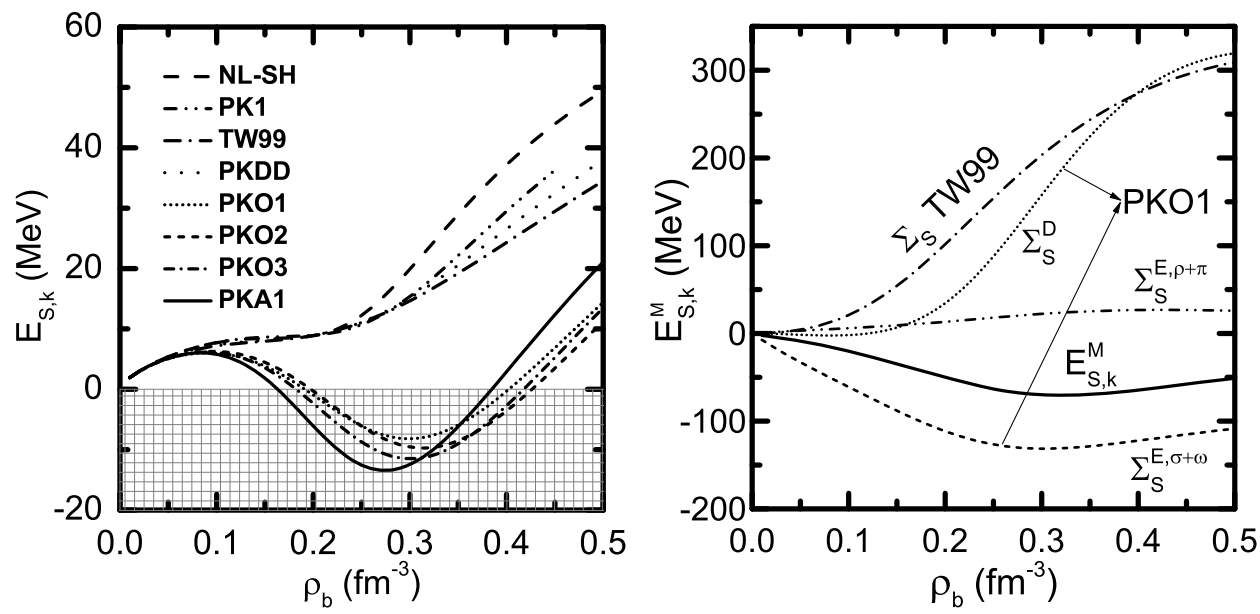

Figure 1: Left panel: the kinetic energy part $E_{S, k}$ of the nuclear symmetry energy as a function of baryonic density $\rho_{b}$. The results are calculated with RHF models PKO1, PKO2, PKO3 and PKA1, in comparison with RMF ones NL-SH, PK1, TW99 and PKDD. Right panel: the mass-related term $E_{S, k}^{M}$ of the kinetic symmetry energy is separated according to various components of the self-energy, see Eq.(4) for details. The results are calculated with RHF model PKO1, in comparison with RMF one TW99.

To identify the physical mechanism, $E_{S, k}$ is further decomposed into the mass- and momentum-related parts according to Eq. (3), namely, $E_{S, k}^{M}$ and $E_{S, k}^{P}$, and the deviations between RMF and RHF results of $E_{S, k}$ are actually dominated by the mass-related term $E_{S, k}^{M}$ [47]. Such model deviations could be further clarified in the right panel of Fig. 1, where the contributions to $E_{S, k}^{M}$ from different components of the scalar self-energy $\Sigma_{S}$ are extracted according to Eq. (4). It is found that the direct terms of the scalar selfenergy $\Sigma_{S}^{D}$ in both RMF and RHF present similar contributions to $E_{S, k}^{M}$. For the Fock terms in PKO1, i.e., $\Sigma_{S}^{E, \sigma+\omega}$ and $\Sigma_{S}^{E, \rho+\pi}$, remarkably negative contribution to $E_{S, k}^{M}$ is found from the isoscalar-meson coupling channel, while relatively weak but still considerable contribution results from the isovector-meson coupling channel, eventually leading to a significant difference in the sign and magnitude of $E_{S, k}$ between RMF and RHF results. In fact, it has been demonstrated very recently that the nuclear tensor in- 
teractions are naturally embedded in both the isoscalar and the isovector meson-nucleon coupling channels with the presence of the Fock diagrams in the RHF EDF $[38,46]$. In consequence, the extra contributions from the Fock terms of $\Sigma_{S}$ to the kinetic symmetry energy could be regarded partly as the effects of the nuclear tensor-force components therein, see Refs. [46, 47].

In short, present status of DDRHF studies on the isospin properties of nuclear matter is summarized, especially the effects of the Fock terms on the nuclear symmetry energy such as those from the hyperon and the nuclear tensor force. The reduction of the kinetic symmetry energy at supranuclear density in DDRHF models is discussed as well, which is related to the fact of a remarkably negative contribution from the isoscalar-meson coupling channel via Fock terms. The results manifest the importance of the Fork diagram in the CDF theory, essentially from the isoscalar-meson coupling channels, on the isospin properties of the in-medium nuclear force.

\section{Acknowledgements}

This work is partly supported by the National Natural Science Foundation of China under Grants No. 11205075 and No. 11375076, and the Specialized Research Fund for the Doctoral Program of Higher Education under Grants No. 20120211120002 and No. 20130211110005.

\section{References}

[1] Brown B. A., Phys. Rev. Lett., 85 (2000) 5296.

[2] Lattimer J. M. and Prakash M., Phys. Rep., 333 (2000) 121.

[3] Baran V. et al., Phys. Rep., 410 (2005) 335.

[4] Steiner A. W. et al., Phys. Rep., 411 (2005) 325.

[5] Li B. A., Chen L. W. and Ko C. M., Phys. Rep., 464 (2008) 113.

[6] Tamii A. et al., Phys. Rev. Lett., 107 (2011) 062502.

[7] Newton W. G. et al., Astrophys. J. Lett., 779 (2013) L4.

[8] Li B. A. et al., Eur. Phys. J. A, 50 (2014) 9.

[9] Chen L. W., Ko C. M. and Li B. A., Phys. Rev. C, 72 (2005) 064309. 
[10] Chen L. W., Ko C. M. and Li B. A., Phys. Rev. C, 76 (2007) 054316.

[11] Akmal A. et al., Phys. Rev. C, 58 (1998) 1804.

[12] Dalen V. E., Fuchs C. and Faessler A., Phys. Rev. C, 72 (2005) 065803.

[13] Taranto G. et al., Phys. Rev. C, 87 (2013) 045803.

[14] Lopes L. L. and Menezes D. P., Phys. Rev. C, 89 (2014) 025805.

[15] Serot B. D. and Walecka J. D., Adv. Nucl. Phys., 16 (1986) 1.

[16] Reinhard P. G., Rep. Prog. Phys., 52 (1989) 439.

[17] Ring P., Prog. Part. Nucl. Phys., 37 (1996) 193.

[18] Bender M. et al., Rev. Mod. Phys., 75 (2003) 121.

[19] Vretenar D. et al., Phys. Rep., 409 (2005) 101.

[20] Meng J. et al., Prog. Part. Nucl. Phys., 57 (2006) 470.

[21] Paar N. et al., Rep. Prog. Phys., 70 (2007) 691.

[22] Liang H. Z., Meng J. and Zhou S. G., Phys. Rep., 570 (2015) 1.

[23] Meng J. and Zhou S. G., J. Phys. G, 42 (2015) 093101.

[24] Miller L. D. and Green A. E. S., Phys. Rev. C, 5 (1972) 241.

[25] Walecka J. D., Ann. Phys. (NY), 83 (1974) 491.

[26] Boguta J. and Bodmer A., Nucl. Phys. A, 292 (1977) 413.

[27] Long W. H., Van Giai N. and Meng J., Phys. Lett. B, 640 (2006) 150.

[28] Long W. H. et al., Phys. Rev. C, 76 (2007) 034314.

[29] Long W. H. et al., EPL, 82 (2008) 12001.

[30] Long W. H. et al., Phys. Lett. B, 680 (2009) 428.

[31] Long W. H. et al., Phys. Rev. C, 81 (2010) 024308.

[32] Long W. H. et al., Phys. Rev. C, 81 (2010) 031302(R).

[33] Ebran J. P. et al., Phys. Rev. C, 83 (2011) 064323. 
[34] Lu X. L., Sun B. Y. and Long W. H., Phys. Rev. C, 87 (2013) 034311.

[35] Niu Z. M. et al., Phys. Rev. C, 87 (2013) 037301.

[36] Wang L. J. et al., Phys. Rev. C, 87 (2013) 047301.

[37] Wang L. J. et al., Phys. Rev. C, 87 (2013) 054331.

[38] Jiang L. J. et al., Phys. Rev. C, 91 (2015) 034326.

[39] Liang H. Z. et al., Phys. Rev. Lett., 101 (2008) 122502.

[40] Liang H. Z., Van Giai N. and Meng J., Phys. Rev. C, 79 (2009) 064316.

[41] Liang H. Z., Zhao P. W. and Meng J., Phys. Rev. C, 85 (2012) 064302.

[42] Liang H. Z. et al., Phys. Rev. C, 86 (2012) 021302(R).

[43] Sun B. Y. et al., Phys. Rev. C, 78 (2008) 065805.

[44] Long W. H. et al., Phys. Rev. C, 85 (2012) 025806.

[45] Niu Z. M. et al., Phys. Lett. B, 723 (2013) 172.

[46] Jiang L. J. et al., Phys. Rev. C, 91 (2015) 025802.

[47] Zhao Q., Sun B. Y. and Long W. H., J. Phys. G, 42 (2015) 095101.

[48] Bouyssy A. et al., Phys. Rev. C, 36 (1987) 380.

[49] Vidaña I. et al., Phys. Rev. C, 84 (2011) 062801(R).

[50] Carbone A., Polls A. and Rios A., EPL, 97 (2012) 22001.

[51] Xu C., Li A. and Li B. A., J. Phys. Conf. Ser., 420 (2013) 012090.

[52] Hen O. et al., Phys. Rev. C, 91 (2015) 025803. 